\title{
Oncotype DX breast cancer recurrence score can be predicted with a novel nomogram using clinicopathologic data
}

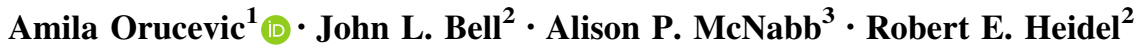

Received: 30 January 2017/ Accepted: 17 February 2017/Published online: 27 February 2017

(C) The Author(s) 2017. This article is published with open access at Springerlink.com

\begin{abstract}
Purpose Oncotype DX (ODX) recurrence score (RS) breast cancer (BC) assay is costly, and performed in only $\sim 1 / 3$ of estrogen receptor (ER)-positive BC patients in the USA. We have now developed a user-friendly nomogram surrogate prediction model for ODX based on a large dataset from the National Cancer Data Base (NCDB) to assist in selecting patients for which further ODX testing may not be necessary and as a surrogate for patients for which ODX testing is not affordable or available.

Methods Six clinicopathologic variables of 27,719 ODXtested ER+/HER2-/lymph node-negative patients with 6-50 $\mathrm{mm}$ tumor size captured by the NCDB from 2010 to 2012 were assessed with logistic regression to predict highrisk or low-risk ODXRS test results with TAILORX-trial and commercial cut-off values; 12,763 ODX-tested patients in 2013 were used for external validation. The predictive accuracy of the regression model was yielded
\end{abstract}

Part of this study was presented at the San Antonio Breast Cancer Symposium in the poster discussion session (PD7-04) on December 9, 2016.

Electronic supplementary material The online version of this article (doi:10.1007/s10549-017-4170-3) contains supplementary material, which is available to authorized users.

Amila Orucevic

aorucevic@utmck.edu

1 Department of Pathology, University of Tennessee Medical Center, 1924 Alcoa Hwy, Knoxville, TN 37920, USA

2 Department of Surgery, University of Tennessee Medical Center, 1924 Alcoa Hwy, Knoxville, TN 37920, USA

3 Graduate School of Medicine, University of Tennessee Medical Center, 1924 Alcoa Hwy, Knoxville, TN 37920, USA using a Receiver Operator Characteristic analysis. Model fit was analyzed by plotting the predicted probabilities against the actual probabilities. A user-friendly calculator version of nomograms is available online at the University of Tennessee Medical Center website (Knoxville, TN).

Results Grade and progesterone receptor status were the highest predictors of both low-risk and high-risk ODXRS, followed by age, tumor size, histologic tumor type and lymph-vascular invasion (C-indexes- 0.85 vs. 0.88 for TAILORx-trial vs. commercial cut-off values, respectively).

Conclusion This is the first study of this scale showing confidently that clinicopathologic variables can be used for prediction of low-risk or high-risk ODXRS using our nomogram models. These novel nomograms will be useful tools to help physicians and patients decide whether further ODX testing is necessary and are excellent surrogates for patients for which ODX testing is not affordable or available.

Keywords Oncotype DX breast cancer recurrence score . Nomograms predicting Oncotype DX results .

Clinicopathologic variables predict Oncotype DX score

\section{Introduction}

Oncotype DX (ODX) (Genomic Health, Redwood City, CA) is a commercially available 21-gene breast cancer recurrence score assay, which has both prognostic and predictive value for estrogen receptor-positive $(\mathrm{ER}+) / \mathrm{hu}-$ man epidermal growth factor receptor 2-negative (HER2)/lymph node-negative breast cancer patients [1]. The ODX recurrence score predicts benefit of adding adjuvant chemotherapy to hormonal manipulation [2]. ODX testing 
is currently endorsed by American Society of Clinical Oncology (ASCO), the National Comprehensive Cancer Network (NCCN), and others for routine guideline application [3-5].

ODX is costly, a factor which contributes to the test being performed in only approximately one-third of eligible breast cancer patients in the United States [6]. It is estimated that ODX is used in less than $20 \%$ of patients in European countries due to limited access and reimbursement [7]. Disparities of its use in the United States have recently been published $[6,8-10]$.

A few recent institution-based studies attempted to predict the results of ODX test using a limited number of respective institutions' patients tested with ODX [11-15]. They correlated the results with certain histopathologic variables available from pathology reports such as immunohistochemical expressions of ER and progesterone receptor (PR), immunohistochemical expression or fluorescence in situ hybridization results for HER2, as well as tumor grade, and tumor size [11-15]. A majority of the models also used immunohistochemical expression of Ki67 proliferation index in spite of its controversy in routine testing of breast cancers [16].

The objective of our study is to develop user-friendly nomograms to be used as surrogate prediction models for a high-risk or a low-risk ODX recurrence score test results. The nomogram development is based on the large breast cancer dataset from the National Cancer Data Base (NCDB), using six common and readily available clinicopathologic variables established in clinical practice as prognostic and/or predictive. These variables are: age, tumor size, tumor grade, PR status, lymph-vascular invasion (LVI), and histologic type of breast cancer (four most common types).

\section{Materials and methods}

\section{Patients and pathology variables selection}

The methods published by Iasonos et al. [17] were used to construct nomograms to predict for a high-risk or a lowrisk ODX recurrence score based on the commercial cutoff recurrence score values $(0-17=$ low-risk and 31-100 $=$ high-risk) or Trial Assigning IndiviuaLized Options for Treatment (NCT00310180)-TAILORx clinical trial (TAILORx-trial) cut-off values $(0-10=$ low-risk and $26-100=$ high-risk). Breast cancer patients tested with ODX assay from 2010 to 2012 and with results recorded as a numerical value $(0-100)$ were identified in the NCDB and served as the study cohort.

The NCDB, a clinical oncology database, acquires data from cancer registries from more than 1,500 Commission on Cancer-accredited facilities with estimated capture of $70 \%$ of all newly diagnosed malignancies in the United States [18]. The NCDB de-identified data regarding the names of patients and institutions prior to the release of the data files. Since criteria of 45 CFR 46.102 d research were met, Institutional Review Board approval was not required.

Inclusion criteria for creation of the nomograms were: (1) female, (2) invasive breast carcinoma, (3) ER positive, (4) HER2 negative, (5) no regional lymph node metastasis, and (6) tumor size between $6 \mathrm{~mm}$ and $50 \mathrm{~mm}$. Our inclusion criteria are the same as the ones recommended as eligibility criteria for ODX testing by the newest 2016 ASCO clinical practice guidelines [3]. Patients also were required to have one of the four most frequent histologic types of breast carcinoma: invasive ductal, invasive lobular, invasive ductal and lobular, or invasive ductal carcinoma mixed with other types. Patients with intermediate ODX score results were excluded, since guidelines for the role of adjuvant chemotherapy in this group of patients remains under investigation in an ongoing prospective TAILORx clinical trial.

The outcome of interest for the nomograms was the probability of a high-risk or a low-risk ODX recurrence score.

\section{Nomogram development and statistical methods}

Simultaneous logistic regression models were used to construct the nomograms. Tumor size and age were considered continuous variables, tumor grade and histology were ordinal, and LVI and PR were coded as follows: $0=$ absent $\mathrm{LVI}, 0=\mathrm{PR}$-negative; $1=$ present $\mathrm{LVI}$, $1=$ PR-positive. The outcome variable was also similarly coded with $0=$ low-risk ODX recurrence score as a reference category and $1=$ high-risk ODX recurrence score as the outcome of interest when predicting for a high-risk ODX recurrence score. Coded outcome variables were reversed when predicting for a low-risk ODX recurrence score. Multicollinearity among the predictor variables was assessed using the variance inflation factor (VIF). VIF values at or above 2.5 assumed evidence of multicollinearity in a model. Calibration of the model was checked by plotting the predicted probabilities against the actual probabilities. Discrimination of the model was assessed using a receiver operating characteristic (ROC) curve to yield a concordance index (c-index) with 95\% confidence interval $(95 \% \mathrm{CI})$.

In order to validate the original model, a second independent sample of patients was collected from the NCDB from breast cancer patients tested with ODX assay in 2013, and with results recorded as a numerical value $(0-100)$. The logistic regression model was performed with the same predictor and outcome variables. Multicollinearity, 
calibration, and discrimination of the validation model were assessed using the same methods. The findings of the original model and the validation model were then compared.

In order to create a nomogram from the predicted probabilities, a scoring system from 0 to 100 was created. To calculate scores based on possessing predictor characteristics, the beta coefficients $(\beta)$ for each predictor variable were given numerical point values. The predictor variable with the highest $\beta$ was assigned 100 points. Then, each remaining $\beta$ was ranked, divided by the highest $\beta$, and multiplied by 100 to yield their respective point values. For age and tumor size, absolute maximum $\beta$ values were assigned by multiplying the raw $\beta$ value by the range for age or tumor size, respectively. The point values for each variable in the model were summed and linked to their respective probabilities of having a high-risk or a low-risk ODX recurrence score. Larger values denoted a higher probability of having a high-risk or a low-risk ODX recurrence score. All statistical analyses were conducted using SPSS Version 22 (Armonk, NY: IBM Corp.), and then confirmed in R program (Vienna, Austria) [19-21].

An easy, user-friendly online nomogram calculator was developed in order to expedite the calculations of the probability of a low-risk or a high-risk ODX score for each patient and is available at the University of Tennessee Medical Center web site: https:/gsm.utmck.edu/ nomograms.

\section{Results}

The original cohort of patients captured by the NCDB between 2010 and 2012 consisted of 27,685 ODX-tested patients when applying commercial cut-off values for a low-risk or a high-risk recurrence score. The external validation cohort consisted of 12,763 ODX-tested patients captured by the NCDB in 2013. Descriptive characteristics of these patients with six clinicopathologic variables chosen for a nomogram creation are contrasted in Tables 1 and 2. Age, tumor size, and PR were found to be statistically significantly different when the original cohort was compared to the external validation cohort of patients $(<.05)$. However, these difference were not considered clinically significant.

In multivariate logistic regression analysis, age, tumor size, tumor grade, PR status, LVI, and histologic tumor type were significantly associated with a low-risk or a highrisk ODX recurrence score test result (Online Resource tables A1 through A4). The tumor grade and PR status showed the highest odds ratios: for example, grade 1 tumor was 49.42 times more likely to be associated with a low- risk recurrence score than a grade 3 tumor $(95 \% \mathrm{CI}$ 41.37-59.03, $p<.001$, commercial cut-off values); negative PR was 0.052 times less likely to be associated with a low-risk recurrence score $(95 \%$ CI .046-.059, $p<.001$; Table A1). In Appendix online only Tables A1 through A4, the results of the final logistic regression analysis of six clinicopathologic variables used for creation of the nomograms are listed including $\beta$ values.

Four nomograms were developed based on these analyses in the original cohort group ( $n=27,685$ for commercial ODX cut-off values; $n=15,623$ for TAILORXtrial ODX cut-off values; points assigned for nomograms shown in Online Resource Tables A5 and A6. There was no evidence of multicollinearity found in our model. The overall predictive accuracy of the model (c-index) measured by the ROC curve was .887 (95\% CI .880-.893) for commercial ODX cut-off recurrence score values and .851 (95\% CI .845-.857) for TAILORx-trial cut-off values.

Four nomograms in the external validation group were developed ( $n=12,685$ for commercial ODX cut-off values; $n=7454$ for TAILORx-trial ODX cut-off values) and are shown in Figs. 1, 2, 3, and 4; points assigned for nomograms are shown in Online Resource Tables A5 and A6. Source code is presented in Online Resource document A1. There was no evidence of multicollinearity. The overall predictive accuracy of the model (c-index) measured by the ROC curve was .89 (95\% CI .88-.90) for commercial ODX cut-off values and .852 (95\% CI .842.861) for TAILORX-trial ODX cut-off values.

It was established that from six chosen clinicopathologic variables, tumor grade and PR status had the highest significant impact on predicting a high-risk or a low-risk ODX recurrence score with either commercial cut-off values or TAILORx-trial cut-off values, followed by age, histologic tumor type and tumor size, while LVI had minimal impact (Figs. 1, 2, 3 and 4 and Online Resource Tables A5 and A6).

\section{Utilizing the nomograms}

Each nomogram (Figs. 1, 2, 3, and 4) consists of nine rows. For an individual patient, each of the six variables is assigned point values based on their clinicopathologic characteristics (topmost scale; see also Online Resource Tables A5 and A6 for assigned points). A vertical line is drawn between the variable value and the topmost "points" line. All allotted points for six variables are added, and the total is found in the "total points" row. A vertical line is then drawn between the final "total points" row and the "predicted probability" row, assigning the final predicted probability for a high-risk or a low-risk ODX recurrence score results for an individual patient. 


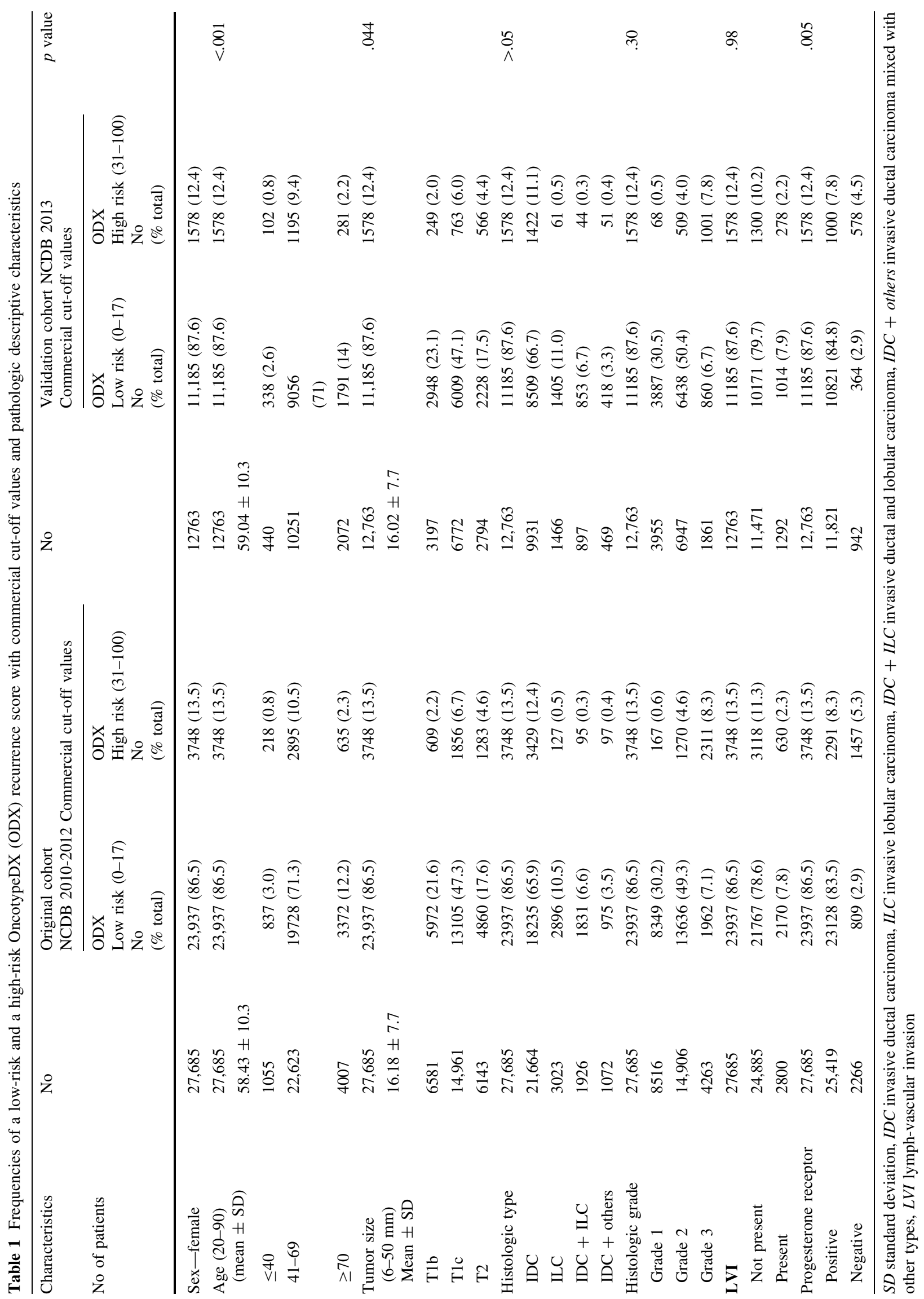




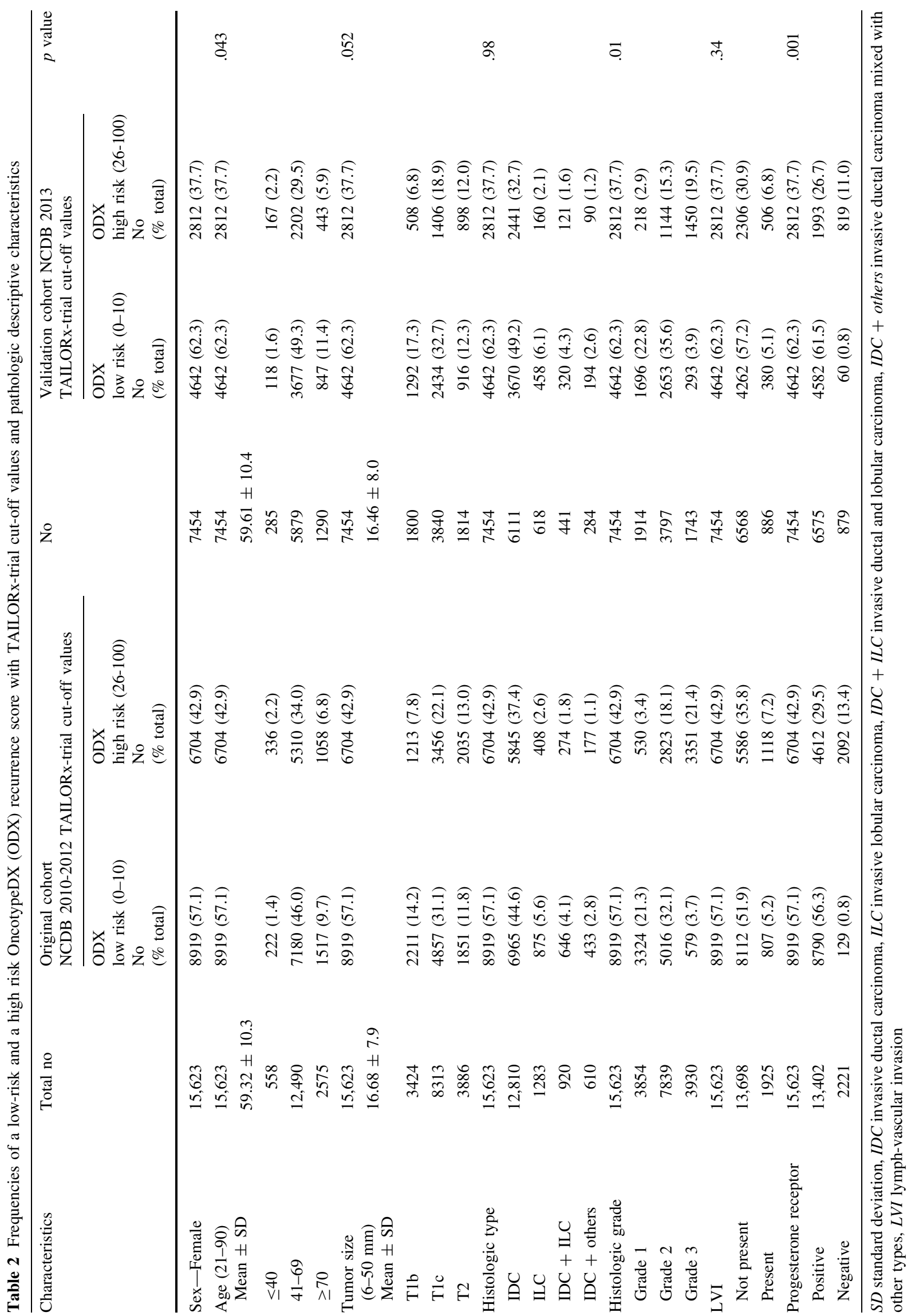




\begin{tabular}{|c|c|c|c|c|c|c|c|c|c|c|c|}
\hline & 0 & 10 & 20 & 30 & 40 & 50 & 60 & 70 & 80 & 90 & 100 \\
\hline
\end{tabular}

Age

$90 \quad 80 \quad 70 \quad 60 \begin{array}{lllllll}1 & 50 & 40 & 30 & 20\end{array}$

Tumor Size

\begin{tabular}{|c|c|c|c|}
\hline $\begin{array}{ll}5 & 10\end{array}$ & 20 & 30 & 40 \\
\hline
\end{tabular}

Histologic Grade

2

$\longdiv { 1 } 3$

Progesterone Receptor

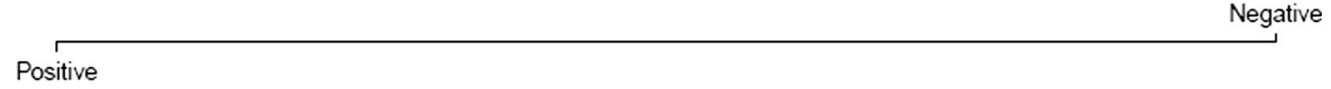

LVI

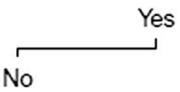

Histologic Type

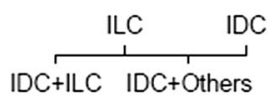

Total Points

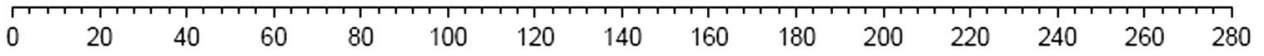

Predicted High Score

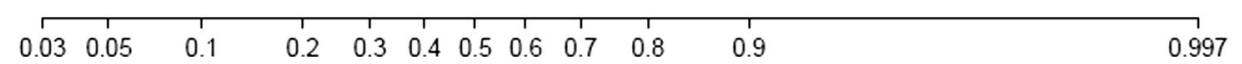

Fig. 1 Nomogram to predict for high-risk Oncotype DX (ODX) score (TAILORx-trial cut-off values 26-100). IDC invasive ductal carcinoma, ILC invasive lobular carcinoma, IDC $+I L C$ invasive

Nomograms from the external validation cohort were used for development of our user-friendly online nomogram calculator in order to expedite the calculations of the probability of a low-risk or a high-risk ODX score for each patient (https://gsm.utmck.edu/nomograms).

\section{Discussion}

ODX was the most commonly utilized breast cancer genomic assay in the United States recorded by the NCDB in the time period 2010-2012: from 202,075 ER+/HER2negative/lymph node-negative breast cancer patients, 69,415 (34\%) had genomic tests performed with $97 \%$ of tests being ODX. ODX is expensive (the current estimated cost is $\sim \$ 4000$ [22]), a factor which contributes to the test being performed in only approximately one-third of breast cancer patients in the USA [6] and in $<20 \%$ of patients in European countries [7]. Several recent institution-based studies performed on a limited number of patients have ductal and lobular carcinoma, IDC + others invasive ductal carcinoma mixed with other types

used some of the pathologic variables routinely available from pathology reports in order to predict ODX test results [11-13, 15, 23-27]. Some of these studies have suggested that ordering ODX test in certain cases would not significantly contribute to clinical management decisions [11, 15, 27]. However, all of these studies were performed on a limited number of patients (institution based). A couple of studies used "H-score" for ER and PR scoring system $[12,27]$ which is more prone to interobserver variability. Kim et al. used a different low-risk and highrisk scoring system [15] in which a low-risk recurrence score incorporated TAILORx-trial low and intermediate recurrence scores $(\leq 25)$, and a high score represented TAILORx-trial high-risk recurrence score $(\geq 26)$ [28]. The majority of these studies also used Ki-67 proliferation index, in spite of the lack of consensus on scoring [16], which is also recognized by the newest $8^{\text {th }}$ edition of the American Joint Commission on Cancer (AJCC), which will be in use from January 2018 [29]. Ki-67 proliferation index has only "AJCC Level of Evidence: III" in the new AJCC edition. 


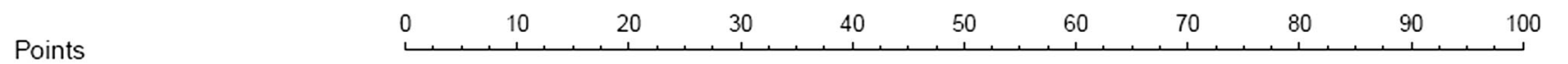

Age

$20 \begin{array}{llllllll} & 30 & 40 & 50 & 60 & 70 & 80 & 90\end{array}$

Tumor Size

Histologic Grade

Progesterone Receptor

LVI

Histologic Type

Total Points

Predicted Probability

$50 \quad 40 \quad 30 \quad 30 \quad 10 \quad 5$

$3+1$
Negative
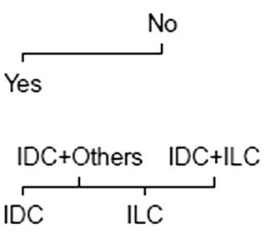

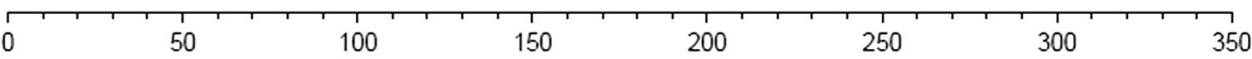

$\begin{array}{lllllllll}0.0027 & 0.01 & 0.05 & 0.1 & 0.25 & 0.5 & 0.75 & 0.9 & 0.967\end{array}$

Fig. 2 Nomogram to predict for a low-risk Oncotype DX (ODX) score (TAILORx-trial cut-off values $0-10$ ). IDC invasive ductal carcinoma, ILC invasive lobular carcinoma, IDC $+I L C$ invasive

We have developed novel, user-friendly nomograms as a surrogate prediction model for ODX test based on the large dataset from the NCDB of ODX-tested ER +/HER2-/lymph node-negative breast cancer patients. We used age, tumor size, tumor grade, PR status, LVI, and the four most common histologic types of breast cancer for predicting ODX test results. These six clinicopathologic variables were established in clinical practice as prognostic and/or predictive and are available from any pathology report. We found that tumor grade and PR status carried the highest predictive value for a low-risk or a high-risk ODX score, which is concordant with results reported by Gage et al. [11] in 540 patients gathered from three different institutions. Finding the grade as the highest predictor for ODX score is not surprising, since the grade was recognized as a significant predictor of breast cancer prognosis many years ago by the Nottingham Prognostic Index $[30,31]$ as well as the surrogate of proliferation at the St. Gallen Consensus Conference in 2011 [32]. In addition, high tumor grade and the 21-gene recurrence score were found as significant ductal and lobular carcinoma, IDC + others invasive ductal carcinoma mixed with other types

predictors of distant recurrence in the founding study of ODX test reported by Paik et al. [1].

PR was also a major predictor of a high-risk or a lowrisk ODX recurrence score in our study, confirming recent observation by Chaudhary et al. in which PR-negative status was associated with higher ODX scores [14]. Our study is the first to show the predictive value of histologic tumor type, age, and tumor size to predict ODX test results. Older patients were less likely to have a high-risk score; larger tumors were more likely to have a high-risk score in our study. These observations were not described in the original study of 21-multigene assay [1], or in a recently published study on optimizing the use of gene expression profiling by Kim et al. [15] perhaps due to the relatively small number of tested patients in those studies in comparison to number of patients in our study. LVI had minimal impact on prediction of ODX test results in our model.

Our nomograms can be used for predicting ODX test results with both TAILORx-trial and commercial cut-off score models. These nomograms therefore accommodate 


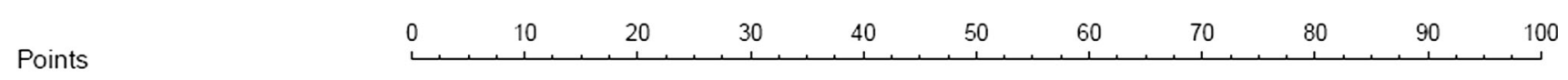

Age

Tumor Size

Histologic Grade

Progesterone Receptor

LVI

Histologic Type

Total Points

Predicted Probability
9072553720

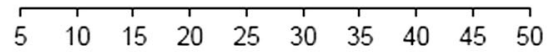

2

1

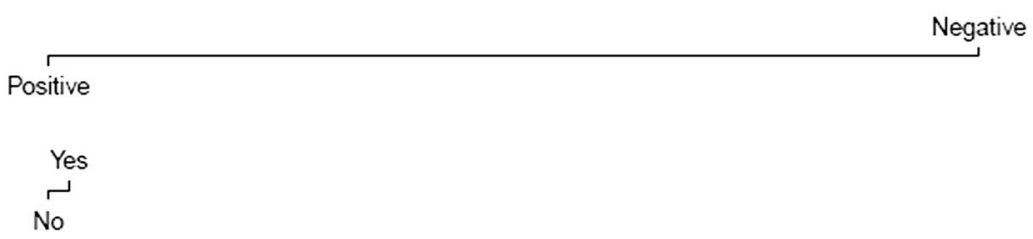

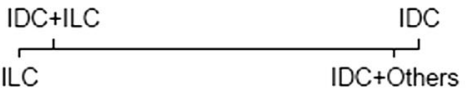

\begin{tabular}{llllllllllllllll}
\hline & 20 & 40 & 60 & 80 & 100 & 120 & 140 & 160 & 180 & 200 & 220 & 240 & 260
\end{tabular}

0.01

$0.05 \quad 0.1$

0.25

0.5

0.75

0.9

0.979

Fig. 3 Nomogram to predict for high-risk Oncotype DX (ODX) score (commercial cut-off values 31-100). IDC invasive ductal carcinoma, ILC invasive lobular carcinoma, $I D C+I L C$ invasive ductal and lobular carcinoma, $I D C+$ others invasive ductal carcinoma mixed with other types

for the differences of ODX scoring results currently in use and allow the interchangeable use of our nomograms/calculators to the clinician's preferences. We believe that these options give our study advantage over other studies which did not use both commercial and TAILORx-trial cut-off values $[11,12,15]$.

ER and PR positivity in our study was established based on the ASCO/CAP guidelines as $\geq 1 \%$ of positive staining cells [33] (guidelines followed by NCDB registrars). ER and $\mathrm{PR}$ status was recorded as either positive or negative in the NCDB without associated levels of positivity. While this could be considered as a weakness of our study, we believe that it was actually a strength, since it confirmed the robustness of our prediction model even without use of additional information such as level of positive expression or the intensity of immunohistochemical staining of ER and PR. Another strength of our study is supported by the large datasets from the National Cancer Data Base. Large number of patients in both the original and the external validation cohorts $(27,685$ and 12,763 , respectively) were used to develop nomograms which had high, acceptable C-indexes (.85-.89). This is the first study of this scale showing confidently that clinicopathologic variables can be used for prediction of low-risk or high-risk ODXRS using our nomogram models.

In the 8th edition of the American Joint Commission on Cancer (AJCC), which will be in use beginning of January 2018, hormone receptor-positive/HER2-negative and lymph node-negative breast cancer patients with a low-risk recurrence score of multigene breast cancer prognostic panels, such as ODX, Mammaprint, EndoPredict, PAM 50, and Breast Cancer Index, have been placed into the same prognostic category as T1a-T1b N0 M0 tumors, regardless of T size. This intent together with the newest 2016 ASCO clinical practice guidelines for use of biomarkers to guide clinical decisions regarding the use of adjuvant systemic therapy for women with early-stage invasive breast carcinoma has embraced the use of genomic prognostic assays as an ideal way of practicing personalized medicine for each breast cancer patient. Unfortunately, these tests are expensive and are not affordable or available for the majority of the breast cancer patients globally. Therefore, 


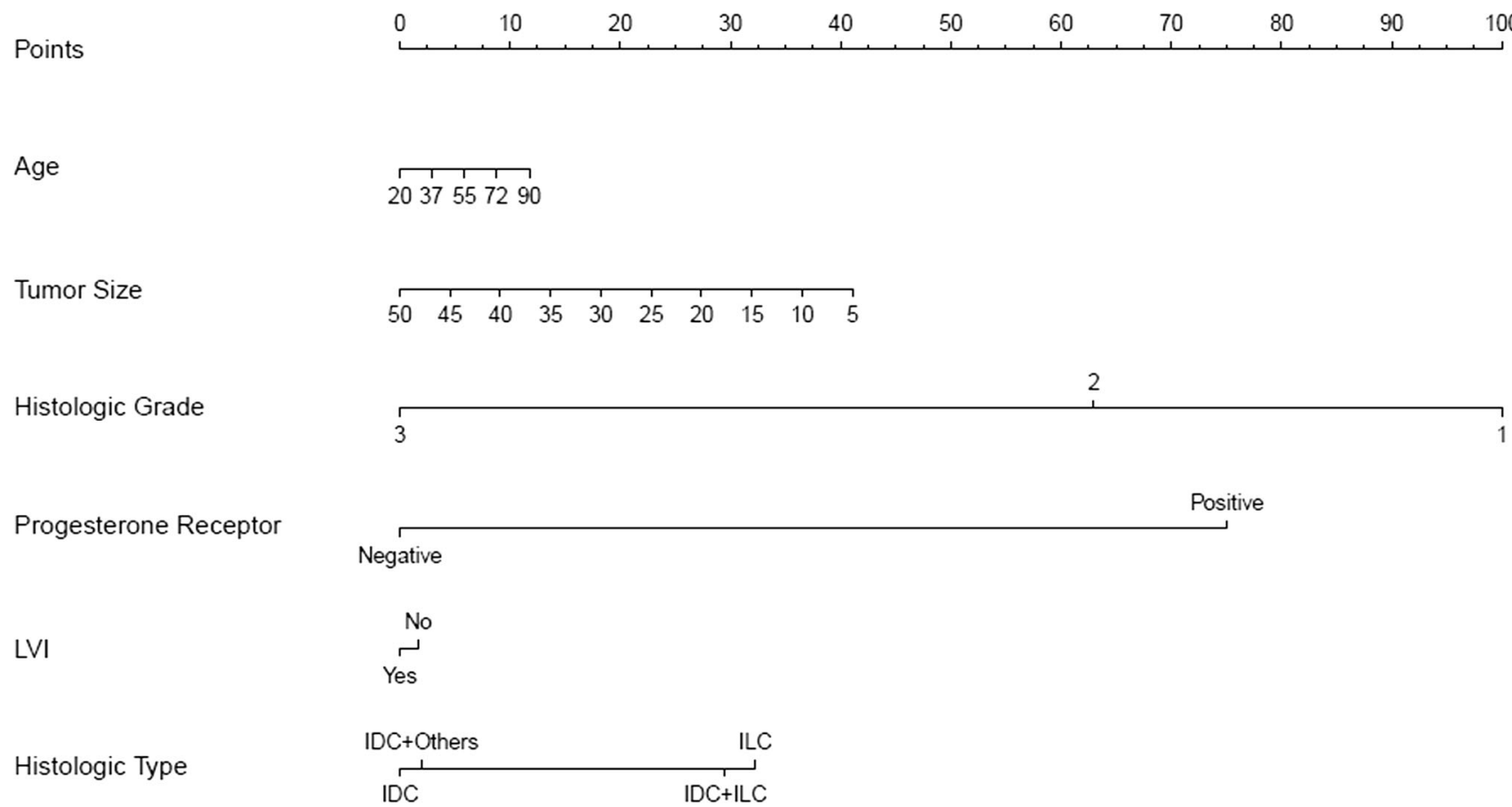

Total Points

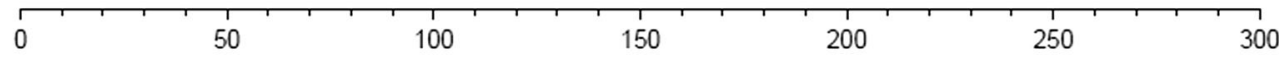

Predicted Probability

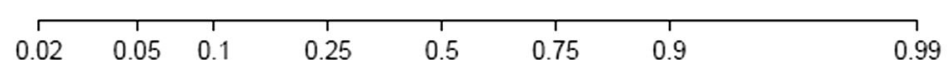

Fig. 4 Nomogram to predict for low-risk Oncotype DX (ODX) score (commercial cut-off values 0-17). IDC invasive ductal carcinoma, ILC invasive lobular carcinoma, $I D C+I L C$ invasive ductal and lobular carcinoma, $I D C+$ others invasive ductal carcinoma mixed with other types

our nomograms which predict for a high-risk or a low-risk ODX recurrence score will be useful tools to assist providers in selecting patients for which further ODX testing may or may not be necessary. They also may serve as a surrogate for patients for which ODX testing is not affordable or available.

Disclaimers The National Cancer Data Base (NCDB) is a joint project of the Commission on Cancer (CoC) of the American College of Surgeons and the American Cancer Society. The CoC's NCDB and the hospitals participating in the CoC NCDB are the source of the deidentified data used herein; they have not verified and are not responsible for the statistical validity of the data analysis or the conclusions derived by the authors.

\section{Compliance with ethical Standards}

Conflict of Interest The authors declare that they have no conflict of interest.

Ethical Approval "All procedures performed in studies involving human participants were in accordance with the ethical standards of the institutional and/or national research committee and with the 1964 Helsinki declaration and its later amendments or comparable ethical standards." For retrospective studies (applies to our study): "For this type of study formal consent is not required".

Ethical Standards The experiments (data analyses) comply with the current laws of the country in which they were performed (United States of America).

Open Access This article is distributed under the terms of the Creative Commons Attribution 4.0 International License (http://crea tivecommons.org/licenses/by/4.0/), which permits unrestricted use, distribution, and reproduction in any medium, provided you give appropriate credit to the original author(s) and the source, provide a link to the Creative Commons license, and indicate if changes were made.

\section{References}

1. Paik S, Shak S, Tang G, Kim C, Baker J, Cronin M, Baehner FL, Walker MG, Watson D, Park T, Hiller W, Fisher ER, Wickerham DL, Bryant J, Wolmark N (2004) A multigene assay to predict recurrence of tamoxifen-treated, node-negative breast cancer. N Engl J Med 351(27):2817-2826. doi:10.1056/NEJMoa041588

2. Paik S, Tang G, Shak S, Kim C, Baker J, Kim W, Cronin M, Baehner FL, Watson D, Bryant J, Costantino JP, Geyer CE Jr, Wickerham DL, Wolmark N (2006) Gene expression and benefit 
of chemotherapy in women with node-negative, estrogen receptor-positive breast cancer. J Clin Oncol 24(23):3726-3734. doi:10.1200/jco.2005.04.7985

3. Harris LN, Ismaila N, McShane LM, Andre F, Collyar DE, Gonzalez-Angulo AM, Hammond EH, Kuderer NM, Liu MC, Mennel RG, Van Poznak C, Bast RC, Hayes DF (2016) Use of biomarkers to guide decisions on adjuvant systemic therapy for women with early-stage invasive breast cancer: American Society of Clinical Oncology Clinical Practice Guideline. J Clin Oncol 34(10):1134-1150. doi:10.1200/jco.2015.65.2289

4. Gradishar WJ, Anderson BO, Balassanian R, Blair SL, Burstein HJ, Cyr A, Elias AD, Farrar WB, Forero A, Giordano SH, Goetz M, Goldstein LJ, Hudis CA, Isakoff SJ, Marcom PK, Mayer IA, McCormick B, Moran M, Patel SA, Pierce LJ, Reed EC, Salerno KE, Schwartzberg LS, Smith KL, Smith ML, Soliman H, Somlo G, Telli M, Ward JH, Shead DA, Kumar R (2015) Breast cancer, version 1.2016. J Natl Compr Canc Netw 13(12):1475-1485

5. Henry NL, Somerfield MR, Abramson VG, Allison KH, Anders CK, Chingos DT, Hurria A, Openshaw TH, Krop IE (2016) Role of patient and disease factors in adjuvant systemic therapy decision making for early-stage, operable breast cancer: American Society of Clinical Oncology Endorsement of Cancer Care Ontario Guideline Recommendations. J Clin Oncol 34(19):2303-2311. doi:10.1200/jco.2015.65.8609

6. Orucevic A, Heidel RE, Bell JL (2016) Utilization and impact of 21-gene recurrence score assay for breast cancer in clinical practice across the United States: lessons learned from the 2010 to 2012 National Cancer Data Base analysis. Breast Cancer Res Treat 157(3):427-435. doi:10.1007/s10549-016-3833-9

7. Albanell J, Svedman C, Gligorov J, Holt SD, Bertelli G, Blohmer JU, Rouzier R, Lluch A, Eiermann W (2016) Pooled analysis of prospective European studies assessing the impact of using the 21-gene Recurrence Score assay on clinical decision making in women with oestrogen receptor-positive, human epidermal growth factor receptor 2-negative early-stage breast cancer. Eur J Cancer (Oxf Eng 1990) 66:104-113. doi:10.1016/j.ejca.2016.06. 027

8. Roberts MC, Weinberger M, Dusetzina SB, Dinan MA, ReederHayes KE, Carey LA, Troester MA, Wheeler SB (2016) Racial variation in the uptake of oncotype DX testing for early-stage breast cancer. J Clin Oncol 34(2):130-138. doi:10.1200/jco.2015. 63.2489

9. Guth AA, Fineberg S, Fei K, Franco R, Bickell NA (2013) Utilization of oncotype DX in an Inner city population: race or place? Int J Breast Cancer 2013:653805. doi:10.1155/2013/ 653805

10. Lund MJ, Mosunjac M, Davis KM, Gabram-Mendola S, Rizzo M, Bumpers HL, Hearn S, Zelnak A, Styblo T, O'Regan RM (2012) 21-Gene recurrence scores: racial differences in testing, scores, treatment, and outcome. Cancer 118(3):788-796. doi:10. $1002 /$ cncr. 26180

11. Gage MM, Rosman M, Mylander WC, Giblin E, Kim HS, Cope L, Umbricht C, Wolff AC, Tafra L (2015) A validated model for identifying patients unlikely to benefit from the 21-gene recurrence score assay. Clin Breast Cancer 15(6):467-472. doi:10. 1016/j.clbc.2015.04.006

12. Klein ME, Dabbs DJ, Shuai Y, Brufsky AM, Jankowitz R, Puhalla SL, Bhargava R (2013) Prediction of the Oncotype DX recurrence score: use of pathology-generated equations derived by linear regression analysis. Mod Pathol 26(5):658-664. doi:10. 1038/modpathol.2013.36

13. Ingoldsby H, Webber M, Wall D, Scarrott C, Newell J, Callagy G (2013) Prediction of Oncotype DX and TAILORx risk categories using histopathological and immunohistochemical markers by classification and regression tree (CART) analysis. Breast (Edinb Scotl) 22(5):879-886. doi:10.1016/j.breast.2013.04.008
14. Chaudhary LN, Jawa Z, Szabo A, Visotcky A, Chitambar CR (2016) Relevance of progesterone receptor immunohistochemical staining to Oncotype DX recurrence score. Hematol/Oncol Stem Cell Therapy 9(2):48-54. doi:10.1016/j.hemonc.2015.12.001

15. Kim HS, Umbricht CB, Illei PB, Cimino-Mathews A, Cho S, Chowdhury N, Figueroa-Magalhaes MC, Pesce C, Jeter SC, Mylander C, Rosman M, Tafra L, Turner BM, Hicks DG, Jensen TA, Miller DV, Armstrong DK, Connolly RM, Fetting JH, Miller RS, Park BH, Stearns V, Visvanathan K, Wolff AC, Cope L (2016) Optimizing the use of gene expression profiling in earlystage breast cancer. J Clin Oncol 34(36):4390-4397

16. CAP (2014) Template for reporting results of biomarker testing of specimens from patients with carcinoma of the breast. http:// www.cap.org/ShowProperty?nodePath=/UCMCon/Contribu tion $\% 20$ Folders/WebContent/pdf/cp-breast-biomarker-template14.pdf. Accessed 26 Dec 2016

17. Iasonos A, Schrag D, Raj GV, Panageas KS (2008) How to build and interpret a nomogram for cancer prognosis. J Clin Oncol 26(8):1364-1370. doi:10.1200/jco.2007.12.9791

18. Cadoo KA, Fornier MN, Morris PG (2013) Biological subtypes of breast cancer: current concepts and implications for recurrence patterns. Quart J Nucl Med Mol Imaging 57(4):312-321

19. R Core Team (2016) R: A language and environment for statistical computing. R Foundation for Statistical Computing. https:// www.R-project.org/.2017

20. Harrell FEJ (2017) rms: regression modeling strategies. http:// CRAN.R-project.org/package $=$ rms

21. Harrell F (2015) Regression modeling strategies: with applications to linear models, logistic and ordinal regression, and survival analysis, 2nd edn. Springer, New York

22. Breastcancer.org Oncotype DX Test. http://www.breastcancer. org/symptoms/testing/types/oncotype_dx. Accessed 1/2/2017 2017

23. Geradts J, Bean SM, Bentley RC, Barry WT (2010) The Oncotype DX recurrence score is correlated with a composite index including routinely reported pathobiologic features. Cancer Invest 28(9):969-977. doi:10.3109/07357907.2010.512600

24. Tang $P$, Wang J, Hicks DG, Wang X, Schiffhauer L, McMahon L, Yang Q, Shayne M, Huston A, Skinner KA, Griggs J, Lyman G (2010) A lower Allred score for progesterone receptor is strongly associated with a higher recurrence score of 21-gene assay in breast cancer. Cancer Invest 28(9):978-982. doi:10. 3109/07357907.2010.496754

25. Allison KH, Kandalaft PL, Sitlani CM, Dintzis SM, Gown AM (2012) Routine pathologic parameters can predict Oncotype DX recurrence scores in subsets of ER positive patients: who does not always need testing? Breast Cancer Res Treat 131(2):413-424. doi:10.1007/s10549-011-1416-3

26. Mattes MD, Mann JM, Ashamalla H, Tejwani A (2013) Routine histopathologic characteristics can predict oncotype DX(TM) recurrence score in subsets of breast cancer patients. Cancer Invest 31(9):604-606. doi:10.3109/07357907.2013.849725

27. Turner BM, Skinner KA, Tang P, Jackson MC, Soukiazian N, Shayne M, Huston A, Ling M, Hicks DG (2015) Use of modified Magee equations and histologic criteria to predict the Oncotype DX recurrence score. Mod Pathol 28(7):921-931. doi:10.1038/ modpathol.2015.50

28. Sparano JA, Gray RJ, Makower DF, Pritchard KI, Albain KS, Hayes DF, Geyer CE Jr, Dees EC, Perez EA, Olson JA Jr, Zujewski J, Lively T, Badve SS, Saphner TJ, Wagner LI, Whelan TJ, Ellis MJ, Paik S, Wood WC, Ravdin P, Keane MM, Gomez Moreno HL, Reddy PS, Goggins TF, Mayer IA, Brufsky AM, Toppmeyer DL, Kaklamani VG, Atkins JN, Berenberg JL, Sledge GW (2015) Prospective validation of a 21-gene expression assay in breast cancer. N Engl J Med 373(21):2005-2014. doi:10.1056/ NEJMoa1510764 
29. AJCC (2016) AJCC cancer staging manual, 8th edn. Springer, Chicago

30. Galea MH, Blamey RW, Elston CE, Ellis IO (1992) The Nottingham Prognostic Index in primary breast cancer. Breast Cancer Res Treat 22(3):207-219

31. Todd JH, Dowle C, Williams MR, Elston CW, Ellis IO, Hinton CP, Blamey RW, Haybittle JL (1987) Confirmation of a prognostic index in primary breast cancer. Br J Cancer 56(4):489-492

32. Goldhirsch A, Wood WC, Coates AS, Gelber RD, Thurlimann B, Senn HJ, Panel M (2011) Strategies for subtypes-dealing with the diversity of breast cancer: highlights of the St. Gallen International Expert Consensus on the Primary Therapy of Early Breast Cancer 2011. Annals of Oncology 22(8):1736-1747. doi:10. 1093/annonc/mdr304
33. Hammond ME, Hayes DF, Dowsett M, Allred DC, Hagerty KL, Badve S, Fitzgibbons PL, Francis G, Goldstein NS, Hayes M, Hicks DG, Lester S, Love R, Mangu PB, McShane L, Miller K, Osborne CK, Paik S, Perlmutter J, Rhodes A, Sasano H, Schwartz JN, Sweep FC, Taube S, Torlakovic EE, Valenstein P, Viale G, Visscher D, Wheeler T, Williams RB, Wittliff JL, Wolff AC (2010) American Society of Clinical Oncology/College Of American Pathologists guideline recommendations for immunohistochemical testing of estrogen and progesterone receptors in breast cancer. J Clin Oncol 28(16):2784-2795. doi:10.1200/JCO. 2009.25.6529 